\title{
Conhecimento de auxiliares e técnicos de enfermagem sobre o tratamento da úlcera venosa
}

\section{Nurses' aides and technicians knowledge about venous ulcer treatment}

\author{
Cristiane Costa Reis Silva ${ }^{1}$ • Márcia de Matos da Fonseca²
}

\begin{abstract}
RESUMO
O presente estudo teve como objetivo identificar o conhecimento de auxiliares e técnicos de enfermagem de Unidades Básicas de Saúde e Unidades de Saúde da Família de um distrito de Salvador/BA, Brasil, quanto ao tratamento da úlcera venosa. Trata-se de um estudo exploratório, descritivo, com abordagem qualitativa, realizado através de uma pesquisa de campo. Os dados foram coletados por meio de um questionário semiestruturado, aplicados a 6 auxiliares e 20 técnicos de enfermagem. Para a análise dos dados foi utilizada a técnica de análise de conteúdo proposta por Bardin. A temática do estudo foi dividida em cinco categorias: identificação da úlcera venosa; tratamentos tópicos utilizados para a úlcera venosa; processo de escolha do tratamento; recursos disponíveis na unidade para a realização do curativo; e utilização de soluções antissépticas. Os resultados demonstraram que a população do estudo não possui conhecimento científico suficiente sobre a utilização dos materiais necessários para o tratamento da úlcera venosa, nem sobre quanto a identificação patológica. Diante disso, recomenda-se um maior investimento na educação continuada dos profissionais de enfermagem.
\end{abstract}

Palavras-chave: Úlcera Varicosa; Auxiliares de Enfermagem; Técnicos de Enfermagem; Cuidados de Enfermagem.

\begin{abstract}
The present study aims to identify the knowledge of nurses' aides and licensed practical nurses, who work in Primary Care and Family Health centres of district of Salvador/BA, Brasil, about the treatment of venous ulcer. It is a field research with an exploratory, descriptive, qualitative approach. The data were collected through a semistructured questionnaire, applied to 6 nurses' aides and 20 licensed practical nurses. Data analysis was performed according to Bardin's content analysis. The study was divided into five categories: venous ulcer identification; topical treatments used for venous ulcer; the process treatment choice; resources available for dressing in the primary health centres and using of antiseptic solutions. The results showed that these professionals have not enough scientific knowledge about the use of necessary materials for the venous ulcers treatment and the identification of it. Therefore, it is recommended greater investment in continuing education of these professionals.

Keywords: Varicose Ulcer; Nurses' Aides; Licensed Practical Nurses; Nursing Care.
\end{abstract}




\section{INTRODUÇÃO}

As úlceras venosas são decorrentes da anormalidade das válvulas venosas superficiais ou profundas e podem ocorrer devido à obstrução do retorno venoso superficial ou refluxo do sangue venoso, levando a hipertensão venosa. $O$ aparecimento das úlceras acontece em virtude da oxigenação ineficaz tecidual ${ }^{1}$.

A úlcera venosa é a complicação mais séria da insuficiência venosa crônica, predominante na metade distal dos membros inferiores, principalmente na região do maléolo medial ${ }^{1}$. Acomete mais o gênero feminino, sobretudo a população idosa ${ }^{2}$. Aproximadamente $1 \%$ da população adulta é atingida pela úlcera venosa ativa ou cicatrizada, e em pessoas acima de 65 anos, esse número é superior a $4 \% 3$.

Muitos fatores interferem na qualidade de vida dos pacientes que apresentam úlcera venosa, dentre ele: mau cheiro, dor, exsudação abundante, mobilidade reduzida, diminuição do sono, longo tempo para a cicatrização da úlcera e as trocas frequentes de curativo, levando ao isolamento social,à necessidade de cuidados especializados e à visitas frequentes as unidades de saúde ${ }^{4}$.

Para que haja uma assistência adequada no tratamento da úlcera venosa é preciso que a equipe de enfermagem tenha conhecimento sobre o processo de reparo tecidual, as doenças de base e suas implicações e as características clínicas da úlcera. Fatores como infecção podem prejudicar a cicatrização da ferida e a restauração da pele ${ }^{5}$.

Uma eficaz ferramenta que oferece os cuidados necessários aos portadores de úlcera venosa é a Atenção Básica à Saúde, uma vez que os profissionais visam à assistência integral e com qualidade para a população, realizando o atendimento domiciliar e na unidade, com foco na família².

O curativo na atenção primária é executado com maior predominância pelos técnicos e auxiliares de enfermagem, mas cabe ao enfermeiro avaliar a ferida, orientar e supervisionar a equipe de enfermagem, e prescrever as coberturas necessárias, devido ao domínio da técnica ${ }^{6}$.

$\mathrm{O}$ avanço do conhecimento no tratamento de feridas contribui para que o auxiliar e o técnico de enfermagem, assim como o enfermeiro, busquem novas maneiras de cuidar do paciente, olhando-o como um todo, desde as possíveis patologias, os aspectos psicológicos, as condições socioeconômica, familiar e cultural. Dessa forma é importante que a enfermagem veja o portador de feridas como um ser único e avalie cada caso especificamente ${ }^{7-8}$.

Tendo em vista a importância da enfermagem no tratamento das úlceras venosas, o conhecimento desses profissionais sobre os diversos fatores que envolvem o tratamento desta patologia é imprescindível, pois dessa forma os pacientes terão uma reabilitação bem sucedida e uma vida com mais qualidade.

Por serem os profissionais técnicos e auxiliares de enfermagem os mais atuantes na realização dos curativos nas Unidades de Saúde ${ }^{6}$, surgiu a necessidade de conhecer a realidade assistencial prestada por eles, propondo a seguinte questão da pesquisa: Qual a abordagem terapêutica dos auxiliares e técnicos de enfermagem em relação à úlcera venosa?

Em virtude dos altos índices de prevalência da úlcera varicosa e o impacto na qualidade de vida dos pacientes, considera-se importante o conhecimento desse tema para que o profissional de enfermagem tenha conhecimento na prestação do cuidado. Este estudo tem como objetivo identificar o conhecimento de auxiliares e técnicos de enfermagem de (UBS) e (USF) do distrito de Subúrbio Ferroviário do município de Salvador/BA, Brasil, quanto ao tratamento da úlcera venosa.

\section{MÉTODO}

Trata-se de um estudo exploratório, descritivo, com abordagem qualitativa realizado através de uma pesquisa de campo. O presente estudo foi desenvolvido nas Unidades de Sáude da Família e Unidades Básicas de Saúde do distrito do Subúrbio Ferroviário do município Salvador/BA, Brasil.

O município de Salvador, no âmbito da saúde, apresenta-se dividido em 12 Distritos Sanitários. 0 distrito de Subúrbio Ferroviário dispõe de 17 Unidades de Saúde da Família e 5 Unidades Básicas de Saúde. A escolha desse distrito foi justificada em virtude da Instituição de Ensino Superior possuir vínculos com as unidades de saúde dessa região com a atuação do Programa de Integração de Saúde e Comunidade (PISCO) onde há a participação de seus alunos, incluindo a pesquisadora, na promoção e prevenção a saúde.

A população desta pesquisa foi composta por auxiliares e técnicos de enfermagem. Foram utilizados como critério de inclusão: todos os profissionais auxiliares e técnicos de enfermagem que atuam nas Unidades de Saúde da Família e Unidades Básicas de Saúde pesquisadas, vinculadas ao distrito sanitário do Subúrbio Ferroviário, que estivessem presentes nas unidades no período de coleta de dados e que aceitassem participar do estudo. Não participaram da pesquisa os profissionais de licença ou de férias, os que não estavam presentes no dia da coleta e aqueles que não aceitaram participar do estudo.

Optou-se por realizar esse estudo em 6 Unidades de Saúde que possuem no total de 8 auxiliares e 27 técnicos de enfermagem atuantes. A amostra foi composta por 26 colaboradores, sendo 6 auxiliares e 20 técnicos de enfermagem que aceitaram participar da pesquisa.

A pesquisa foi realizada após obtenção do parecer favorável (CAAE 30782214600005033) do Comitê de Ética em Pesquisa da própria Universidade, via Plataforma Brasil, cumprindo os aspectos éticos de acordo com Resolução 466/2012 do Conselho Nacional de Saúde (CNS). Após a aprovação, foi enviado para a Secretaria Municipal de Saúde 
um pedido de autorização para realização da pesquisa. Os profissionais que aceitaram participar do estudo assinaram o Termo de Consentimento Livre e Esclarecido, após terem sido orientados sobre o total sigilo dos participantes e os objetivos do estudo.

Os dados foram coletados nos meses de fevereiro e março de 2016, por meio de um questionário semiestruturado, aplicado nas Unidades de Saúde, contendo questões fechadas que buscaram traçar o perfil social e de formação profissional dos participantes e, questões abertas que visavam à análise qualitativa proposta nos objetivos da pesquisa. Foi escolhida a técnica de análise de conteúdo proposta por Bardin, dividida nas seguintes etapas: a pré-análise, a exploração do material e o tratamento dos resultados, inferência e interpretação.

\section{RESULTADOS E DISCUSSÕES}

Os profissionais que participaram do estudo foram predominantemente do gênero feminino, $88 \%$ e $12 \%$ do gênero masculino. A enfermagem historicamente é uma profissão exercida em sua grande maioria por mulheres, fato que decorre de uma percepção pelo qual as atividades do cuidado são próprias da natureza biológica das mulheres, seja na criação dos filhos ou na estruturação social das distinções sexuais 9 .

Quanto a idade, os sujeitos de estudo estão na faixa etária de 25 a 56 anos. A maioria (69\%) encontra-se entre a faixa etária de 28 e 41 anos. De acordo com a pesquisa de Ximenes Neto et $a l^{9}$, essa faixa etária também foi predominante e representa uma parcela significativa da população economicamente ativa.

O tempo de formação profissional variou entre 1 a 33 anos. Constituindo a maioria (62\%) entre 4 e 12 anos, com média de 11 anos de atuação. Observa-se que os profissionais possuem bastante tempo de experiência como auxiliar ou técnico de enfermagem. Segundo uma pesquisa realizada por Peduzzi \& Anselm ${ }^{10}$, a experiência prática é vista pelos profissionais da área como critério de desempenho profissional.

Com respeito à realização de cursos de atualização sobre feridas, $27 \%$ dos profissionais afirmaram nunca ter realizado nenhum tipo de capacitação e dentre os que realizaram, 19\% haviam feito o curso há mais de um ano. De acordo com Ximenes Neto et al ${ }^{9}$, muitos auxiliares e técnicos de enfermagem de UBS e USF se sentem inseguros na realização de algumas ações de enfermagem, sentido inclusive necessidade de aperfeiçoamento. O Ministério da Saúde com a implementação da Ação Permanente em Saúde, pela portaria $n^{\circ}$ 1.996, de 20 de agosto de 2007, dispõe de uma diretriz que contempla o SUS objetivando a qualificação dos profissionais da área de saúde ${ }^{11}$.

A análise referente a temática do estudo foi dividida em cinco categorias: identificação da úlcera venosa; os tratamentos tópicos utilizados para a úlcera venosa; o processo de escolha do tratamento; recursos disponíveis na unidade para a realização do curativo; e utilização de soluções antissépticas.

Quando questionado aos auxiliares e técnicos de enfermagem quanto a identificação da úlcera venosa, observa-se um desconhecimento das características dessas úlceras por alguns sujeitos (P. 3 e P. 9), que citam a úlcera por pressão e queimadura, respectivamente, em vez da úlcera venosa. E uma escassez dos dados fornecidos para a avaliação da úlcera por outros indivíduos (P.16 e P. 20). Conforme relatos abaixo:

Através do surgimento de pontos de vermelhidão em locais que estejam passando por pressões devido a falta de movimentação do membro ou área afetada. (P. 3).

Vascularização e vermelhidão do local, primeiro aparece bolhas e depois o ferimento. (P. 9).

Uma lesão de difícil cicatrização. (P. 16).

Membros inferiores, aspecto, profundidade, tempo de cicatrização demorada. (P. 20).

Nos depoimentos acima fica evidente a falta de conhecimento dos entrevistados sobre a patologia. $\mathrm{O}$ que se conclui que muitos dos profissionais agem apenas de maneira prática sem se preocupar em ter um embasamento teórico.

Para o diagnóstico clínico da úlcera venosa, os critérios analisados são: a localização da ferida, na parte medial da região inferior da perna; o aspecto do leito da ferida, granuloso e com coloração avermelhada ou com presença de necrose, geralmente esfacelo; as bordas, lisas e irregulares; presença de exsudato, moderada a intensa; pele circundante hiperpigmentada, lipodermatoesclerose, dilatação venosa, veias reticulares, veias varicosas, atrofia branca, edema e dores que são aliviadas com a elevação dos membros inferiores ${ }^{1-12}$.

Porém alguns dos profissionais conseguiram diagnosticar a úlcera venosa, apesar dos indicadores relatados não estarem completos, como evidenciado abaixo:

Localizado nos membros inferiores, a região ao redor fica escurecida e a úlcera apresenta coloração avermelhada. (P. 1).

Tonalidade mais escura da pele e endurecida ao redor da lesão e presença de vasos alterados. (P. 4).

Ferida em região maleolar, hiperpigmentação, alívio da dor com a elevação do membro. (P. 10).

Os sintomas apresentados pelos profissionais são característicos de um paciente portador de úlcera venosa. Foram relatados por eles os seguintes sintomas: hiperpigmentação da pele circundante, localização da ferida, dilatação venosa, lipodermatoesclerose e a dor aliviada com a elevação do membro inferior. 
Sobre a utilização do tratamento tópico pelos auxiliares e técnicos de enfermagem, foram encontrados os seguintes relatos demonstrados abaixo:

Colagenase, hidrogel com ou sem alginato, AGE, gaze vaselinada ao redor da pele. (P. 4).

Colagenase, sulfadiazina de prata, hidrogel e Bota de Unna, porém somente a enfermagem que coloca. (P. 5).

Bota de Unna, hidrogel, alginato, hidrofilme e carvão ativado. (P. 19).

Bota de Unna, carvão ativado, colagenase e sulfadiazina de prata. (P. 23).

Percebe-se que os profissionais não relataram a escolha do curativo conforme as características da ferida, quantidade de exsudato, presença ou ausência de infecção ${ }^{12}$. Alguns profissionais informaram utilizar a Bota de Unna, mas somente $58 \%$ deles, sendo a terapia compressiva de fundamental importância para que a cicatrização da úlcera venosa seja bem sucedida. Uma vez que a compressão age aumentando o volume de ejeção do sangue venoso durante a deambulação ${ }^{3}$.

Segundo Liao, Cheater ${ }^{13}$, o curativo deveria ser de baixo custo e não aderente a fim de evitar danos ao leito da lesão, contudo na presença de tecido inviável é necessário realizar o desbridamento que pode ser autolítico, químico ou mecânico.

O desbridamento autolítico é promovido através da utilização de coberturas oclusivas que mantem o leito da ferida úmida. $O$ desbridamento químico envolve o uso de enzimas, entre elas a colagenase e a papaína. Já o desbridamento mecânico pode ser realizado cirurgicamente ou aplicando curativos úmidos ou secos, esse método pode remover também tecido viável ${ }^{3-12}$. Segundo Sellmer et $a l^{4}$, o desbridamento nas úlceras com necrose e esfacelos utiliza-se o hidrogel ou hidrocóloide ou papaína diluída a $10 \%$ ou gel a $1 \%$.

Há várias coberturas disponíveis no mercado, o seu uso varia de acordo com as características da úlcera venosa, dentre as mais conhecidas estão: o hidrocolóide utilizado em úlcera com pouco a moderado exsudato; o alginato de cálcio em úlcera com moderado a intenso exsudato; espuma de poliuterano em úlcera dolorosa e com moderada a grande exsudação; úlcera com necrose é indicado o hidrogel; úlcera com moderada a alta exsudação e infectada indica-se espuma de poliuretano com prata ou carvão ativado que possui a propriedade de eliminar odores desagradáveis ${ }^{3-4}$.

Se a pele ao redor da úlcera estiver seca ou escamosa recomenda-se o uso do AGE; os filmes semipermeáveis possuem pouca capacidade de absorver exsudato, por isso não é recomendado no tratamento da úlcera venosa-14. Segundo um estudo realizado por Abdalla, Dadalti ${ }^{15}$, em quatro meses houve uma redução do tamanho da ferida e formação de tecido de granulação após a utilização da sulfadiazina de prata e do nitrato de cério na úlcera venosa.

Em relação a escolha do tratamento tópico, os auxiliares e técnicos de enfermagem mencionaram os seguintes relatos:

A escolha depende da avaliação de uma enfermeira da unidade que é especializada em feridas. (P. 2).

Indicação médica ou enfermeiro responsável pelo curativo. (P. 7).

De acordo com a extensão da ferida, as características da lesão e com o que disponibilizamos de material no momento para realização do mesmo. (P. 13).

Não tem escolha, aqui na unidade é o que tiver na hora. (P. 17).

Percebe-se que os profissionais (P.2 e P.7) estão corretos ao relatarem que eles não são os responsáveis pela escolha do tratamento tópico, sendo o enfermeiro o responsável pelos critérios de escolha. Quanto aos relatos dos profissionais (P.13 e P.17), observa-se que não há critério escolha, nem mesmo pelo enfermeiro da unidade, apenas utilizam dos materiais disponíveis no momento.

Avaliar e tratar uma ferida é responsabilidade da equipe de enfermagem, assim sendo é necessário que o profissional de enfermagem realize o diagnóstico preciso quanto ao tipo de lesão para que haja a escolha correta do produto no tratamento da úlcera venosa, visto que seu uso inadequado pode prejudicar ou retardar a cicatrização ${ }^{8-16}$.

Os recursos mais comuns encontrados na sala de curativo de acordo com os profissionais são: Bota de Unna, clorexidina, sulfadiazina de prata, colagenase e hidrogel.

Quanto ao emprego das soluções antissépticas na úlcera venosa, $63 \%$ dos entrevistados mencionaram a clorexidina, $37 \%$ dos entrevistados informaram realizar a limpeza somente com SF 0,9\%.

O SF 0,9\% por ser uma solução isotônica e possuir o mesmo PH do plasma é altamente recomendável na limpeza de feridas, pois ele não altera a microbiota natural da pele. Além disso SF 0,9\% não interfere no processo de cicatrização, não causa danos tecidual e não provoca reações alérgicas ${ }^{14}$.

Em feridas altamente infectadas as soluções antissépticas podem ser utilizadas, uma vez que auxiliam na prevenção da infecção e também apresenta vantagens em relação aos antibióticos tópicos, pois não originam bactérias resistentes às drogas e possuem taxas mais baixas de sensibilização ${ }^{17}$.

Apesar do efeito citotóxico da clorexidina, em feridas infectadas o seu uso favorece a cicatrização tecidual devido a sua propriedade antibacteriana ${ }^{18}$. Recentemente um antisséptico de baixa toxidade para os tecidos vem sendo bastante utilizado na limpeza de feridas, o hidrocloropolihexametilenobiguanida (PHMB). Eficaz no tratamento de feridas crônicas e/ou infectadas, reduzindo o processo 
infeccioso e inflamatório, além de acelerar o tempo de cicatrização, não provoca dor na aplicação, irritabilidade cutânea ou alergias ${ }^{19}$.

Em uma pesquisa realizada por Moscati et al ${ }^{20}$, os autores revelaram o benefício do uso da água de torneira na limpeza de feridas. A água de torneira contém insuficiente número de bactérias que possam provocar uma infecção à ferida e também não são patógenas para pele. Outra vantagem é a alta pressão de irrigação no leito da ferida que favorece a remoção de pequenas partículas de matérias e bactérias da lesão e o seu baixo custo.

\section{CONCLUSÃO}

Pode-se perceber a partir dos dados analisados que os profissionais não possuem conhecimento científico suficiente sobre a utilização dos materiais necessários para o tratamento da úlcera venosa, nem sobre a identificação desta patologia. Sem esse conhecimento é muito difícil realizar o diagnóstico correto da lesão e indicar o produto necessário para o tratamento da lesão, portanto a qualidade dos serviços prestados ao cliente e a recuperação do tecido lesionado são prejudicadas.

Assim, diante dos resultados apresentados fica evidente a necessidade de uma qualificação profissional. Recomenda-se então um maior investimento na educação continuada dos auxiliares e técnicos da Atenção Primária, a fim de garantir uma atuação humanizada e com qualidade.

\section{REFERÊNCIAS}

1. Oliveira BGRB, Nogueira GA, Carvalho MR, Abreu AM. Caracterização dos pacientes com úlcera venosa acompanhados no Ambulatório de Reparo de Feridas. Rev Eletrônica Enferm. [internet] 2012;14(1):156-63 [acessado 2016 Mar 15]. Disponível em: https://revistas.ufg.emnuvens. com.br/fen/article/view/10322/0.

2. Martins DA, Souza AM. O perfil dos clientes portadores de úlcera varicosa cadastrados em programas de saúde pública. Cogitare Enferm. 2007;12(3):353-7.

3. Abbade, LPF, Lastoria, S. Abordagem de pacientes com úlcera da perna de etiologia venosa. An Bras Dermatol. 2006;81(6):509-22.

4. Sellmer D, Carvalho CMG, Carvalho DR, Malucelli, A. Sistema especialista para apoiar a decisão na terapia tópica de úlceras venosas. Rev. Gaúcha Enferm. 2013;34(2).

5. Brandão ES. Enfermagem em dermatologia: cuidados técnico, dialógico e solidário. Rio de Janeiro: Cultura médica; 2006.

6. Morais GFC, Oliveira SHS, Soares MJGO. Avaliação de feridas pelos enfermeiros de instituições hospitalares da rede pública. Texto Contexto Enferm. 2008;17(1): 98-105.

7. Carneiro, CM, Sousa, FB, Gama FN. Tratamento de feridas: assistência de enfermagem nas unidades de atenção primária à saúde. Revista Enfermagem Integrada. 2010;3(2).

8. Pereira AL, Bachion MM. Tratamento de feridas: análise da produção científica publicada na Revista Brasileira de Enfermagem de 1970-2003. Rev Bras Enferm. 2005;58(2).
9. Ximenes Neto FRG, Costa MCF, Rocha J, Cunha ICKO. Auxiliares e técnicos de enfermagem na saúde da família: perfil sociodemográfico e necessidades de qualificação. Trab Educ Saúde. 2008;6(1):51-64.

10. Peduzzi M,Anselmi ML. O auxiliar e o técnico de enfermagem: categorias profissionais diferentes e trabalhos equivalentes. Rev Bras Enferm. 2004;57(4): 425-9.

11. Brasil. Ministério da saúde. Portaria $n^{\circ} 1.996$, de 20 de agosto de 2007. Dispõe sobre as diretrizes para a implementação da Política Nacional de Educação Permanente em Saúde. Diário Oficial da República Federativa do Brasil. 236 nov. 2007. Seção 1, p. 140.

12. Hess CT. Tratamento de feridas e úlceras. 4. ed. Rio de Janeiro: Reichmann \& Affonso Editores; 2002. p. 109-27.

13. Liao XH, Cheater F. The management of patients with venous leg ulcers. London: Audit Protocol; 2000. p.14.

14. Borges EL. Tratamento tópico de úlcera venosa: proposta de uma diretriz baseada em evidências [tese]. Ribeirão Preto (SP): Universidade de São Paulo; 2005.

15. Abdalla S, Dadalti P. Uso da sulfadiazina de prata associada ao nitrato de cério em úlceras venosas: relato de dois casos. An Bras Dermatol. 2003;78 (2).

16. Solomé MS. Avaliando lesão: práticas e conhecimentos dos enfermeiros que prestam assistência ao indivíduo com ferida. Physis. 2009;35(6):280-7.

17. Drosou A, Falabella A, Kirsner RS, Antiseptics on wounds: an area of controversy. Health Management Publications. 2003; 15(5):149-66.

18. Cavalcanti Neto AT, Arruda TEP, Arruda TTP, Pereira SLS, Turati E. Análise comparativa entre o óleo-resina de copaíba e o digluconato de clorexidina no processo de cicatrização tecidual: estudo histológico em dorso de ratos. Rev Odontol UNESP. 2005; 34(2): 107-12.

19. Santos EJF, Silva MANCGMM. Tratamento de feridas colonizadas / infetadas com utilização de polihexanida. Rev Enf Ref. 2011; 3(4):135-42.

20. Moscati RM, Mayrose J, Reardon RF, Janicke DM, Jehle DV. A multicenter comparison of tap water versus sterile saline for wound irrigation. Acad Emerg Med. 2007;14(5):404-9. 


\section{APÊNDICE A - QUESTIONÁRIO}

Identificação (iniciais):

Idade:

Gênero: ( ) Feminino ( ) Masculino

Há quanto tempo você atua como auxiliar ou técnico de enfermagem:

Já fez curso de atualização sobre feridas? ( ) Sim ( ) Não

Quando foi seu último curso de atualização sobre feridas?

Como você identifica uma úlcera venosa?

Quais os tipos de tratamento tópico que você utiliza na úlcera venosa?

Quais os recursos disponíveis para realização do curativo na unidade?

Como se dá a escolha do tratamento?

Quais as soluções antissépticas que você conhece para a úlcera venosa e que você utiliza? 


\section{APÊNDICE B - COMITÊ DE ÉTICA EM PESQUISA TERMO DE CONSENTIMENTO LIVRE E ESCLARECIDO.}

Resolução nº 466/2012 - Conselho Nacional de Saúde

O Sr (a) está sendo convidado (a) a participar voluntariamente da pesquisa intitulada "Conhecimento dos auxiliares e técnicos de enfermagem sobre o tratamento da úlcera venosa", que tem como objetivo identificar o conhecimento dos auxiliares e técnicos de enfermagem das Unidades Básicas de Saúde e Unidades de Saúde da Família do município de Salvador/BA quanto o tratamento da úlcera venosa a fim de proporcionar uma melhor reabilitação desses pacientes.

A coleta de informações será por meio de um questionário e serão tratadas de forma anônima e sigilosa. Ressaltamos ainda que o Sr. (Sra.) poderá desistir da pesquisa em qualquer momento, sem que the seja imputada penalidades ou prejuízos e nenhuma compensação financeira. A sua participação contribuirá para aumentar o conhecimento da ciência na área da enfermagem.

Nome da Orientadora: Cristiane Costa Reis Silva

E-mail: cristianereisfb@gmail.com

Telefone: (071) 99368-0001

Nome do Orientado: Márcia de Matos da Fonseca

E-mail: marcia1880@hotmail.com

Telefone: (071) 99187-2222

$\mathrm{Eu}$, concordo em participar deste estudo após as informações supracitadas. Autorizo a utilização dos dados por mim fornecidos para a realização da pesquisa. Tenho conhecimento do seu caráter cientifico, sendo minha participação voluntaria. Estou ciente de que as informações fornecidas terão o anonimato e o sigilo, e de que não sofrerei nenhum tipo de sansão ou prejuízo caso decida, a qualquer momento, desistir de participar do estudo.

Salvador-BA, de de 2016.

(Assinatura do Participante) 